\title{
Epidermal cGVHD Score 4
}

National Cancer Institute

\section{Source}

National Cancer Institute. Epidermal cGVHD Score 4. NCI Thesaurus. Code C158595.

A score of 4 describing severe disease with confluent erythema and scale and/or papules covering $>80 \%$ of target area. 